Editor's Note: Toolboxes are a new, occasional feature in the Journal designed to briefly highlight a new method or a resource of general use in neuroscience or to critically analyze existing approaches or methods. For more information, see http://www. jneurosci.org/misc/itoa.shtml.

\title{
Quantum Dot Applications to Neuroscience: New Tools for Probing Neurons and Glia
}

\author{
Smita Pathak, ${ }^{1}$ Elizabeth Cao, ${ }^{2}$ Marie C. Davidson, ${ }^{3}$ Sungho Jin, ${ }^{1}$ and Gabriel A. Silva ${ }^{1,2,3,4}$ \\ ${ }^{1}$ Materials Science and Engineering Program, Departments of ${ }^{2}$ Bioengineering and ${ }^{3}$ Ophthalmology, and ${ }^{4}$ Neurosciences Program, University of California, \\ San Diego, La Jolla, California 92037-0946
}

Key words: glia; immunocytochemistry; neurons; nanotechnology; quantum dots; imaging

\section{Introduction}

Semiconductor fluorescent quantum dots are nanometer-sized functionalized particles that display unique physical properties that make them particularly well suited for visualizing and tracking molecular processes in cells using standard fluorescence microscopy (Jaiswal et al., 2003; Watson et al., 2003; Michalet et al., 2005). They are readily excitable and have broad absorption spectra with very narrow emission spectra, allowing multiplexing of many different colored quantum dots; they display minimal photobleaching, thereby allowing molecular tracking over prolonged periods; they also display a blinking property that allows the identification of individual quantum dots. As a result, single molecule binding events can be identified and tracked using optical fluorescence microscopy, allowing the pursuit of experiments that are difficult or not possible given other experimental approaches. Neuroscience-specific applications of quantum dots are starting to emerge. Some work has focused on using this nanotechnology to address cellular and molecular questions of interest, al-

Received Sept. 11, 2005; revised Dec. 5, 2005; accepted Dec. 17, 2005

This work was supported by a Culpeper Biomedical Pilot Initiative Award and the Stein Institute for Research on Aging. We thank Quantum Dot for providing the quantum dot kits used in our protocol.

Correspondence should be addressed to Dr. Gabriel A. Silva, University of California, San Diego, Jacobs Retina Center 0946, 9415 Campus Point Drive, La Jolla, CA 92037-0946. E-mail: gsilva@ucsd.edu.

DOI:10.1523/JNEUROSCI.3847-05.2006

Copyright $\odot 2006$ Society for Neuroscience $\quad$ 0270-6474/06/261893-03\$15.00/0 though other work is pushing the development of the technology forward.

Quantum dots are nanometer-sized particles composed of a heavy metal core, such as cadmium selenium or cadmium telluride with an intermediate unreactive zinc sulfide shell and a customized outer coating of different bioactive molecules tailored to a specific application (Fig. 1). The composition and very small size of quantum dots $(5-8 \mathrm{~nm})$ gives them unique and very stable fluorescent optical properties that are readily tunable by changing their physical composition or size. The photochemical properties of quantum dots allow selective fluorescent tagging of proteins similar to classical immunocytochemistry. Additionally, the use of quantum dots is associated with minimal photobleaching and a much higher signal-to-noise ratio. Their broad absorption spectra but very narrow emission spectra allows multiplexing of many quantum dots of different colors in the same sample, something that cannot be achieved with traditional fluorophores. The physics responsible for these effects are beyond the scope of this brief introduction, but the small size of quantum dot particles results in large but specific energy jumps between the energy band gaps of excited electron-hole pairs in the semiconductor core. This effect results in scaled changes of absorption and emission wavelengths as a function of particle size, so that small changes in the radius of quantum dots translate into very distinct changes in color (Arya et al., 2005; Van- maekelbergh and Liljeroth, 2005). This physical property represents another major advantage over traditional organic fluorophores that in general require distinct chemistries to produce different colors. For biological applications, quantum dots can be chemically functionalized to target proteins at high ligand-receptor densities. Recent work has shown that, at least in some cellular systems, quantum dots conjugated with natural ligands are readily internalized into cells, do not interfere with intracellular signaling, and are nontoxic (Chan et al., 2002; Murphy, 2002; Jain, 2003; Watson et al., 2003; West and Halas, 2003).

\section{Quantum dots and neuroscience}

Quantum dots represent a new tool of significant potential in neuroscience research. In addition to offering an alternative to traditional immunocytochemistry, they are particularly valuable for studies of neurons and glia. Quantum dots can be used to visualize, measure, and track individual molecular events using fluorescence microscopy, and they provide the ability to visualize and track dynamic molecular processes over extended periods (e.g., from seconds to many minutes). These properties are difficult to achieve using other techniques or approaches. For example, quantum dots are useful for experiments that are limited by the restricted anatomy of neuronal and glial interactions, such as the small size of the synaptic cleft, or between an astrocyte process and a neuron. Because of their ex- 
tremely small size and optical resolution, they are also well suited for tracking the molecular dynamics of intracellular and/or intercellular molecular processes over long time scales. However, it should be appreciated that the hydrodynamic radius of functionalized quantum dots is larger $(15-20 \mathrm{~nm})$ than their actual size of 5-8 nm (Larson et al., 2003). Recent studies using quantum dots in neuroscience illustrate the potential of this technology. Triller and colleagues (Dahan et al., 2003) used antibody functionalized quantum dots to track the lateral diffusion of glycine receptors in cultures of primary spinal cord neurons. They were able to track the trajectory of individual glycine receptors for tens of minutes at spatial resolutions of 5-10 nm, demonstrating that the diffusion dynamics varied depending on whether the receptors were synaptic, perisynaptic, or extrasynaptic. Vu et al. (2005) tagged nerve growth factor $(\beta N G F)$ to quantum dots and used them to promote neuronal-like differentiation in cultured pheochromocytoma 12 (PC12) cells. Ultimately, these approaches could be used to visualize and track functional responses in neurons. However, as with any new technology, there are caveats. For example, $\mathrm{Vu}$ et al. (2005) reported that $\beta$ NGF conjugated to quantum dots had reduced activity compared with free $\beta$ NGF. Other groups are pushing the technology forward and providing new quantum-dotbased tools. Brinker and colleagues (Fan et al., 2005) developed a technique to produce biocompatible water-soluble quantum dot micelles that retain the optical properties of individual quantum dots. These micelles showed uptake and intracellular dispersion in cultured hippocampal neurons. Ting and colleagues (Howarth et al., 2005) are developing a modified quantum dot labeling approach that addresses the relatively large size of antibody-quantum-dot conjugates and the instability of some quantum-dot-ligand interactions. Their technique tags cell surface proteins with a specific peptide (a 15 aa polypeptide called acceptor protein; GLNDIFEAQKIEVWHE) that can be directly biotinylated as a target for streptavidin-conjugated quantum dots. Using this approach, they were able to specifically label and track AMPA receptors on cultured hippocampal neurons.

Ultimately, quantum dot nanotechnologies will require easy-to-use approaches that can be straightforwardly replicated in a typical neurobiology lab. Unfortunately, in our experience, most quantum dot protocols intended for non-

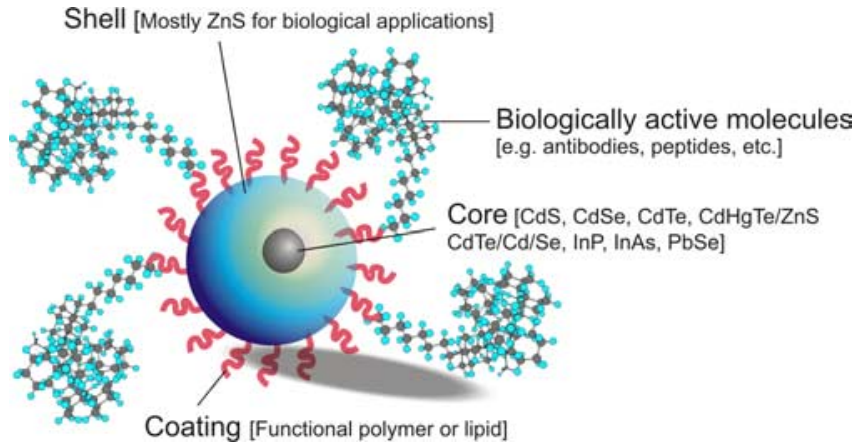

Figure 1. Structure of a semiconductor fluorescent quantum dot nanocrystal. The heavy metal core is responsible for the fluorescence properties of the quantum dot. The nonemissive shell stabilizes the core, whereas the coating layer provides anchor sites to organic and biological ligands such as antibodies, peptides, and other organic molecules.
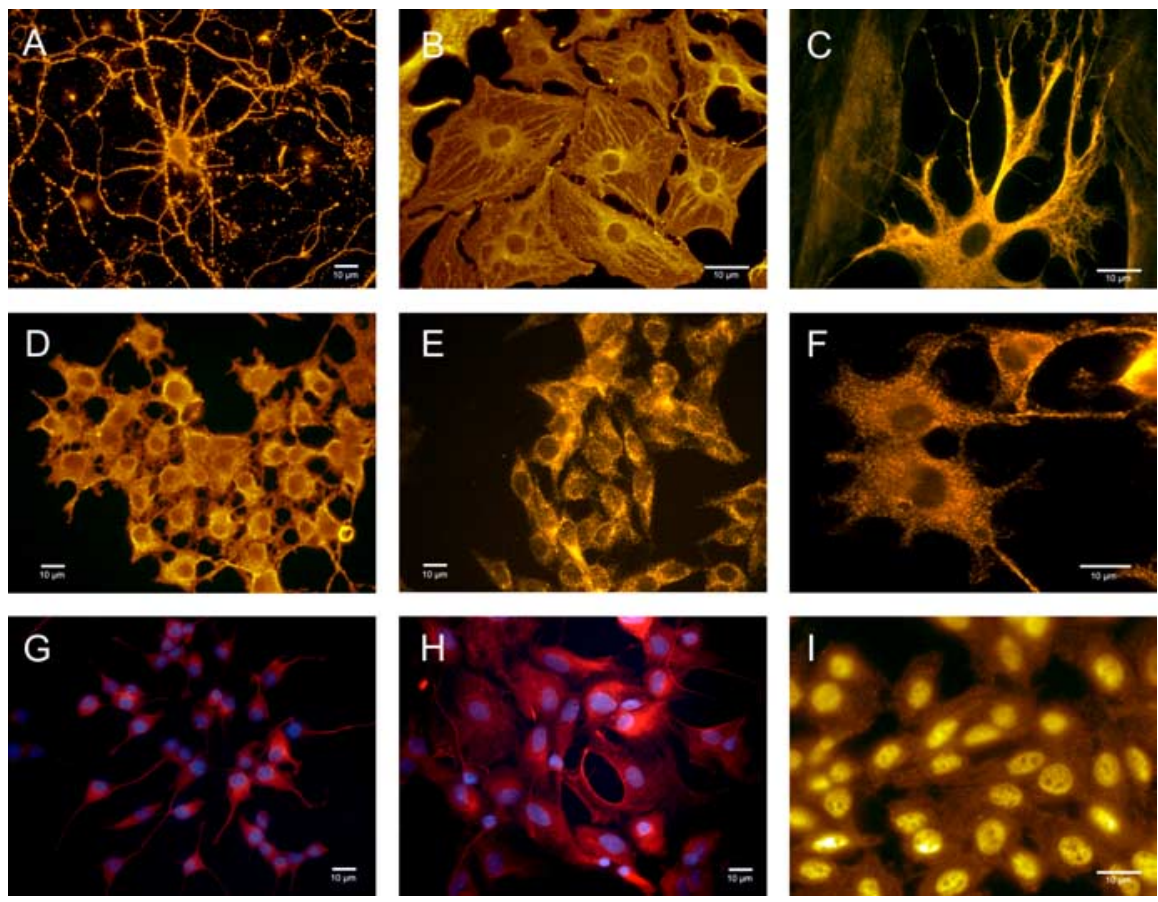

Figure 2. Fluorescent labeling of neurons and glia with antibody-conjugated $605 \mathrm{~nm}$ quantum dots. $\boldsymbol{A}$, Primary cortical neurons specifically labeled for $\beta$-tubulin. $\boldsymbol{B}, \boldsymbol{C}$, Primary cortical astrocytes specifically labeled for glial fibrillary acidic protein (GFAP). D, F, PC12 cells labeled for $\beta$-tubulin. $\boldsymbol{E}$, r-MC1 neural retinal Muller glial cells specifically labeled for GFAP. G, PC12 cells labeled for $\beta$-tubulin using standard immunocytochemistry. $\boldsymbol{H}$, Primary spinal cord astrocytes labeled for GFAP using standard immunocytochemistry. I, An example of artifactual nonspecific labeling in r-MC1 Muller cells with anti-GFAP-conjugated $605 \mathrm{~nm}$ quantum dots. In this case, putative nonspecific electrostatic interactions between quantum dots and cellular proteins led to intense nuclear staining and mild cytoplasmic staining using other quantum dot conjugation protocols described for mammalian cells. All imaging parameters were constant for the different experimental conditions, with an acquisition/exposure time of $30 \mathrm{~ms}$ for all panels, except for $I$, which was taken with an acquisition time of $100 \mathrm{~ms}$.

neural mammalian cells in the peerreviewed literature and in instructions from commercial sources do not always readily work with primary neurons, glia, and related cell lines (Fig. $2 I$ ). In the supplemental data, we present a very simple and straightforward protocol for specific labeling of both neurons and glia (Fig. 2 and supplemental Table 1, available at www.jneurosci.org as supplemental material). Of particular note, as far as we are aware, these results represent the first use of quantum dots in glial cells. Given the unique properties that quantum dots have to offer neuroscience research, their future looks pretty bright (and stable).

\section{References}

Arya H, Kaul Z, Wadhwa R, Taira K, Hirano T, Kaul SC (2005) Quantum dots in bio-imaging: revolution by the small. Biochem Biophys Res Commun 329:1173-1177.

Chan WC, Maxwell DJ, Gao X, Bailey RE, Han M, Nie S (2002) Luminescent quantum dots for multiplexed biological detection and imaging. Curr Opin Biotechnol 13:40-46. 
Dahan M, Levi S, Luccardini C, Rostaing P, Riveau B, Triller A (2003) Diffusion dynamics of glycine receptors revealed by singlequantum dot tracking. Science 302:442-445.

Fan H, Leve EW, Scullin C, Gabaldon J, Tallant D, Bunge S, Boyle T, Wilson MC, Brinker CJ (2005) Surfactant-assisted synthesis of watersoluble and biocompatible semiconductor quantum dot micelles. Nano Lett 5:645-648.

Howarth M, Takao K, Hayashi Y, Ting AY (2005) Targeting quantum dots to surface proteins in living cells with biotin ligase. Proc Natl Acad Sci USA 102:7583-7588.

Jain KK (2003) Nanodiagnostics: application of nanotechnology in molecular diagnostics. Expert Rev Mol Diagn 3:153-161.
Jaiswal JK, Mattoussi H, Mauro JM, Simon SM (2003) Long-term multiple color imaging of live cells using quantum dot bioconjugates. Nat Biotechnol 21:47-51.

Larson DR, Zipfel WR, Williams RM, Clark SW, Bruchez MP, Wise FW, Webb WW (2003) Water-soluble quantum dots for multiphoton fluorescence imaging in vivo. Science 300:1434-1436.

Michalet X, Pinaud FF, Bentolila LA, Tsay JM, Doose S, Li JJ, Sundaresan G, Wu AM, Gambhir SS, Weiss S (2005) Quantum dots for live cells, in vivo imaging, and diagnostics. Science 307:538-544.

Murphy CJ (2002) Optical sensing with quantum dots. Anal Chem 74:520A-526A.
Vanmaekelbergh D, Liljeroth P (2005) Electronconducting quantum dot solids: novel materials based on colloidal semiconductor nanocrystals. Chem Soc Rev 34:299-312.

Vu TQ, Maddipati R, Blute TA, Nehilla BJ, Nusblat L, Desai TA (2005) Peptide-conjugated quantum dots activate neuronal receptors and initiate downstream signaling of neurite growth. Nano Lett 5:603-607.

Watson A, Wu X, Bruchez M (2003) Lighting up cells with quantum dots. Biotechniques 34:296-300, 302-303.

West JL, Halas NJ (2003) Engineered nanomaterials for biophotonics applications: improving sensing, imaging, and therapeutics. Annu Rev Biomed Eng 5:285-292. 